\title{
Stabilizer information inequalities from phase space distributions
}

\author{
David Gross ${ }^{1}$ and Michael Walter ${ }^{2}$ \\ ${ }^{1)}$ Institute for Physics, University of Freiburg, Rheinstrasse 10, 79104 Freiburg, Germany a) \\ ${ }^{2)}$ Institute for Theoretical Physics, ETH Zurich, Wolfgang-Pauli-Str. 27, 8093 Zurich, \\ Switzerland b]
}

(Dated: February 27, 2013; revised: June 13, 2013)

\begin{abstract}
The Shannon entropy of a collection of random variables is subject to a number of constraints, the best-known examples being monotonicity and strong subadditivity. It remains an open question to decide which of these "laws of information theory" are also respected by the von Neumann entropy of many-body quantum states. In this article, we consider a toy version of this difficult problem by analyzing the von Neumann entropy of stabilizer states. We find that the von Neumann entropy of stabilizer states satisfies all balanced information inequalities that hold in the classical case. Our argument is built on the fact that stabilizer states have a classical model, provided by the discrete Wigner function: The phase-space entropy of the Wigner function corresponds directly to the von Neumann entropy of the state, which allows us to reduce to the classical case. Our result has a natural counterpart for multi-mode Gaussian states, which sheds some light on the general properties of the construction. We also discuss the relation of our results to recent work by Linden, Ruskai, and Winter. 1
\end{abstract}

\section{INTRODUCTION AND RESULTS}

The Shannon entropy of a discrete random variable $X$ is given by $H(X)=-\sum_{x} p_{x} \log p_{x}$, where $p_{x}$ is the probability that $X=x$. Given a collection of random variables $X_{1}, \ldots, X_{n}$, we can consider the joint entropy $H\left(X_{I}\right)$ of any non-empty subset $X_{I}=\left(X_{i}\right)_{i \in I}$ of the variables. These entropies are not independent-they are subject to a number of linear homogeneous inequalities, known as information inequalities, or as the "laws of information theory". 2 Conversely, the set of all such inequalities determines the set of possible joint entropies $\left(H\left(X_{I}\right)\right)$ up to closure ${ }^{3}$ There are two classes of fundamental or Shannon-type inequalities. The first is monotonicity, stating that the entropy does not decrease if more random variables are taken into account: $H\left(X_{I \cup J}\right)-H\left(X_{I}\right) \geq 0$. The second class is strong subadditivity,

$$
H\left(X_{I}\right)+H\left(X_{J}\right)-H\left(X_{I \cap J}\right)-H\left(X_{I \cup J}\right) \geq 0 .
$$

Since the seminal work of Zhang and Yeung it is known that there are other entropy inequalities which are not implied by

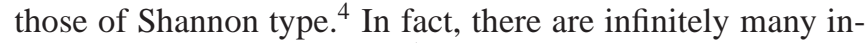
dependent such inequalities $\stackrel{5}{.}$

In quantum mechanics, the state of a quantum state of $n$ particles is described by a density operator $\rho$ on a tensor-product Hilbert space. The state of any subset $I \subseteq\{1, \ldots, n\}$ of the particles is described by the reduced state $\rho_{I}=\operatorname{tr}_{I^{c}} \rho$ formed by tracing out the Hilbert space of the other particles. The natural analogue of the Shannon entropy is the von Neumann entropy $S(\rho)=-\operatorname{tr} \rho \log \rho, \underline{6}$ and it is of fundamental interest to determine the linear inequalities satisfied by the entropies $S\left(\rho_{I}\right)$ of subsystems ${ }^{7}$ The most immediate difference to the classical case is that the von Neumann entropy is no longer monotonic: global quantum states can exhibit less entropy

\footnotetext{
a) Electronic mail: www.qc.uni-freiburg.de

b)Electronic mail: mwalter@itp.phys.ethz.ch
}

than their reductions (a signature of entanglement). Instead, the von Neumann entropy satisfies weak monotonicity:

$$
S\left(\rho_{I \cup K}\right)+S\left(\rho_{J \cup K}\right)-S\left(\rho_{I}\right)-S\left(\rho_{J}\right) \geq 0 .
$$

Strong subadditivity, however, famously remains valid for quantum entropies. ${ }^{8}$ It is a major open problem in quantum information theory to decide whether there are any entropy inequalities beyond the ones given above (see Refs. 9 and 10 for some partial progress, including a class of so-called constrained inequalities).

Strong subadditivity is tight for product states (resp. for independent random variables). An entropy inequality $\sum_{I} \nu_{I} S\left(\rho_{I}\right) \geq 0$ has that property if and only if $\sum_{I \ni i} \nu_{I}=0$ for all $i$. Such an inequality is called balanced (also correlative ${ }^{11}$ or homogeneous ${ }^{1}$ ), and it has been shown that the classical entropy cone is determined by the set of balanced information inequalities together with monotonicity (which is not balanced) $\stackrel{12}{*}$ Balanced entropy inequalities will play an important role below.

Of course, entropy inequalities are also relevant in the case of continuous variables (also classically, see e.g. Ref. 12) as well as for other kinds of entropies, e.g. Rényi entropies. ${ }^{13}$

In this work we study the entropy inequalities satisfied by two classes of quantum states-namely, stabilizer states and Gaussian states (which are the continuous-variable counterpart of the former). These states are versatile enough to exhibit intrinsically quantum features (such as multi-particle entanglement), but possess enough structure to allow for a concise and computationally efficient description. In both cases, quantum phase-space methods have been built around them, and it is this point of view we aim to exploit here. For $n$ systems of dimension $d$, the phase space is $\mathbb{Z}_{d}^{2 n}$, while for $n$ bosonic modes it is given by $\mathbb{R}^{2 n}$. In both cases, it is the direct sum of the single-particle/single-mode phase spaces.

The starting point for our work is the Wigner function, which for Gaussian states as well as for stabilizer states in odd dimensions $d$ is a bona fide probability distribution on the classical phase space (the case of even $d$ requires some 
more care, see Theorem 2). We may thus define random variables $X_{1}, \ldots, X_{n}$ on the phase space, jointly distributed according to the Wigner function of the given quantum state $\rho$. Here, $X_{i}$ denotes the component in the single-particle space of the $i$-th particle or mode. The random variables $X_{1}, \ldots, X_{n}$ constitute our classical model. This construction is compatible with reduction: the marginal probability distribution of a subset of variables $X_{I}=\left(X_{i}\right)_{i \in I}$ is given precisely by the Wigner function of the reduced quantum state $\rho_{I}$. Our crucial observation then is that certain quantum entropies are simple functions of the corresponding classical entropies. More precisely, we find that

$$
S_{2}\left(\rho_{I}\right)=H_{2}\left(X_{I}\right)-C|I|,
$$

where $C>0$ is a universal constant and where $S_{2}(\rho)=$ $-\log \operatorname{tr} \rho^{2}$ and $H_{2}(X)=-\log \sum_{x} p_{x}^{2}$ denote the quantum and classical Rényi-2 entropy, respectively. In the case of continuous variables, we use the differential Rényi entropy $H_{2}(X)=-\log \int p_{x}^{2} d x$. Therefore, if $\sum_{I} \nu_{I} H_{2}\left(X_{I}\right) \geq 0$ is a balanced entropy inequality satisfied by the random variables $X_{I}$ then the same inequality is satisfied by the quantum state,

$$
\sum_{I} \nu_{I} S_{2}\left(\rho_{I}\right)=\sum_{I} \nu_{I} H_{2}\left(X_{I}\right)-C \underbrace{\sum_{I} \nu_{I}|I|}_{=0} \geq 0 .
$$

In the case of stabilizer states (Section $\Pi$ ), all reduced states $\rho_{I}$ are normalized projectors (onto the corresponding code subspace), while the $X_{i}$ are uniformly distributed (on their support). Thus all Rényi entropies agree with each other, and also with the Shannon and von Neumann entropy, respectively:

$$
S\left(\rho_{I}\right)=H\left(X_{I}\right)-|I| .
$$

As above, it follows that any balanced entropy inequality that is valid for the Shannon entropies of the $X_{I}$ is also valid for the von Neumann entropies of the stabilizer states $\rho_{I}$. In particular, stabilizer states respect all balanced information inequalities, such as the inequalities of non-Shannon type found in Ref. 4. What is more, our construction can also be understood in the group-theoretical framework of Ref. 14. Here it is well-known that there are inequalities which do not hold for arbitrary random variables, but only for random variables constructed from certain classes of subgroups, e.g. normal subgroups. ${ }^{15}$ Since phase spaces are Abelian groups, it follows that the von Neumann entropies of stabilizer states also respect such information laws, e.g. the Ingleton inequality,,$\frac{15}{5}$ which is the balanced inequality

$$
I_{\rho}(I: J \mid K)+I_{\rho}(I: J \mid L)+I_{\rho}(K: L)-I_{\rho}(I: J) \geq 0 .
$$

Here, $I_{\rho}(I: J)=S\left(\rho_{I}\right)+S\left(\rho_{J}\right)-S\left(\rho_{I \cup J}\right)$ and $I_{\rho}(I:$ $J \mid K)=S\left(\rho_{I \cup K}\right)+S\left(\rho_{J \cup K}\right)-S\left(\rho_{K}\right)-S\left(\rho_{I J K}\right)$ are the quantum (conditional) mutual information.

We find it instructive to understand how the above classical model manages to respect monotonicity, while the quantum state may violate it. For example, since stabilizer states can be entangled (even maximally so), $H\left(\rho_{1}\right)=H\left(\rho_{2}\right)=1$ and $H\left(\rho_{12}\right)=0$ are perfectly valid entropies of a stabilizer state which obviously violate monotonicity. Equation (2) states that the classical model is more highly mixed than the quantum one, in the sense that the entropy associated with a subset $I$ is higher by an amount of $|I|$. That is precisely the amount by which quantum mechanics can violate monotonicity.

In the case of Gaussian states (Section $\amalg$, the random variables $X_{1}, \ldots, X_{n}$ have a multivariate normal distribution, and we show that the differential Rényi-2 entropy in (1) can be replaced by the Rényi- $\alpha$ entropy for arbitrary positive $\alpha \neq 1$ :

$$
S_{2}\left(\rho_{I}\right)=H_{\alpha}\left(X_{I}\right)-|I|\left(\log \pi-\frac{\log \alpha}{1-\alpha}\right),
$$

In the limiting case $\alpha \rightarrow 1$, we recover a formula involving the differential Shannon entropy which has previously appeared in Ref. 16, attributed to Stratonovich. Thus, Rényi-2 entropies of Gaussian states respect all balanced information inequalities that hold for multivariate normal distributions $\frac{17,18}{\mathrm{In}-}$ terestingly, it is not clear whether a similar statement holds for the von Neumann entropy of the Gaussian state. This is perhaps an indication that the connection (2) between the Shannon and the von Neumann entropy for stabilizer states is somewhat coincidental. The comparison with Gaussian states suggests that the Rényi-2 entropies might be the more fundamental quantities in this context, that merely happen to agree with the von Neumann entropy in the case of stabilizer states.

We conclude this section with a few remarks. Our work uses the classical model provided by the Wigner function as a tool for proving statements that do not, a priori, seem to be connected to phase space distributions. This point of view has been employed before, e.g. to construct quantum expanders,, 19 to establish simulation algorithms, $\stackrel{20-22}{=}$ and for demonstrating the onset of contextuality. $\stackrel{23}{=}$ It would be interesting to see further applications.

While it is known that the Wigner function approach cannot be straight-forwardly translated to non-stabilizer states, $\stackrel{24-26}{26}$ our discussion suggests searching for other maps from quantum states to probability distributions that reproduce entropies faithfully, up to state-independent additive constants.

In order to establish the Ingleton inequality (3), we have used the group-theoretical approach to classical information inequalities $\stackrel{14}{=}$ It would be highly desirable to find a quantummechanical analogue of this work (see Refs. 27 and 28 for partial results towards this goal, motivated by the quantum marginal problem of quantum physics).

Related Work. Independently of this work, Linden, Ruskai, and Winter have published an analysis of the entropy cone generated by stabilizer states. $\stackrel{1}{-}$ Their methods - focusing on group-theoretical constructions - are conceptually complementary to our phase-space approach. Ref. 1 contains a complete characterization of the entropy cone generated by fourparty stabilizer states. The paper also lists further example of inequalities which, like the Ingleton Inequality, are respected by stabilizer states, even though there are classical distributions violating it. While not stated explicitely, their methods 
can readily be used to prove that all balanced inequalities remain valid for stabilizers (see Theorem 11 in Ref. 1 and discussion thereafter).

Convention. In this work, entropies of $d$-level systems are measured in units of $\log d$ bits. In the continuous-variable case, we employ the natural logarithm.

\section{STABILIZER STATES}

In this section, we describe our results on stabilizer states. We start by fixing some notation and recalling the basic formalism of stabilizer states $\stackrel{6.29}{=}$ The phase-space methods that we employ work most smoothly over Hilbert spaces of odd dimension and therefore the exposition is focused on that case. However, discrete phase spaces and stabilizer states can be defined for any dimension $d$ and our main result is valid in full generality. Theorem 1 summarizes the precise statements that we require to prove our results, and we present a selfcontained account of the general theory in Appendix A We then establish our main result - a classical model for the von Neumann entropy of stabilizer states - in Theorem 2

Let $d>1$ be an odd integer. The discrete configuration space of a single particle is $\mathbb{Z}_{d}$, where $\mathbb{Z}_{d}=\mathbb{Z} / d \mathbb{Z}$ is the set of congruence classes modulo $d$. The associated phase space is the $\mathbb{Z}_{d}$-module $\mathbb{Z}_{d}^{2}=\mathbb{Z}_{d} \oplus \mathbb{Z}_{d}$. We denote the components of "vectors" $v \in \mathbb{Z}_{d}^{2}$ by $(p, q)$ in order to emphasize the analogy with momentum and position in the continuous-variable theory. A crucial piece of structure is the symplectic form defined on the phase space. It maps vectors $v=(p, q)$ and $v^{\prime}=\left(p^{\prime}, q^{\prime}\right)$ to $\left[v, v^{\prime}\right]=p q^{\prime}-q p^{\prime}$. For each vector $v=(p, q)$, we define a Weyl operator acting on the Hilbert space of complex functions on $\mathbb{Z}_{d}$, which can be identified with $\mathbb{C}^{d}$ : It is given by $(w(p, q) \psi)(x)=e^{\frac{2 \pi \mathrm{i}}{d}\left(p x-2^{-1} p q\right)} \psi(x-q)$, where $2^{-1}:=\left(d^{2}+1\right) / 2$ denotes a multiplicative inverse of 2 modulo $d$ (this only exists for odd $d$ ). A direct calculation shows that

$$
w(v) w\left(v^{\prime}\right)=e^{\frac{2 \pi \mathbf{i}}{d} 2^{-1}\left[v, v^{\prime}\right]} w\left(v+v^{\prime}\right) .
$$

Thus the Weyl operators realize a projective or twisted representation of the additive group of the phase space $\mathbb{Z}_{d}^{2}$ (it is a faithful representation of the Heisenberg group over $\mathbb{Z}_{d}$, see e.g. Ref. 30).

For $n$ particles, the phase space is the direct sum $V=$ $\bigoplus_{i=1}^{n} V_{i}=\mathbb{Z}_{d}^{2 n}$ of the single-particle phase spaces $V_{i}=\mathbb{Z}_{d}^{2}$. It can be represented on $\left(\mathbb{C}^{d}\right)^{\otimes n}$ by the tensor product of the single-particle representations, $w(v)=\bigotimes_{i=1}^{n} w\left(v_{i}\right)$, and the composition law (4) continues to hold if we extend the symplectic form linearly.

Let us now consider an isotropic submodule $M \subseteq V$, i.e. a submodule on which the symplectic form vanishes. Isotropicity implies by (4) that the Weyl operators $\{w(m): m \in M\}$ form an Abelian group-a stabilizer group. One easily verifies that

$$
P(M):=\frac{1}{|M|} \sum_{m \in M} w(m)
$$

defines an orthogonal projection onto a $d^{n} /|M|$-dimensional subspace of $\left(\mathbb{C}^{d}\right)^{\otimes n}$. This subspace is called the stabilizer code associated with $M$; it contains the vectors in $\left(\mathbb{C}^{d}\right)^{\otimes n}$ that are invariant under the stabilizer group. The corresponding stabilizer state is the density operator

$$
\rho(M)=\frac{1}{\operatorname{tr} P(M)} P(M)=\frac{1}{d^{n}} \sum_{m \in M} w(m),
$$

and its von Neumann entropy is given by

$$
S(\rho(M))=n-\log |M| .
$$

One obtains a larger set of stabilizer codes by including certain phase factors in the sum in (5),,$\frac{6,25,29}{}$ However, all stabilizer codes are locally equivalent to one of the form (5). Since we are only interested in the entropy of $\rho(M)$ and of its reduced density matrices, we may therefore safely restrict to the class of stabilizer states defined above.

One obtains a simple expression for the reduced state $\rho(M)_{I}$ by using the relation $\operatorname{tr} w\left(v_{i}\right)=d \delta_{v_{i}, 0}$. For this, let $V_{I}:=\left\{v \in V: v_{i}=0\right.$ for $\left.i \notin I\right\}$ be the phase space of a subset of particles $I \subseteq\{1, \ldots, n\}$, and set $M_{I}:=M \cap V_{I}$. Then $\rho(M)_{I}=\rho\left(M_{I}\right)$, i.e. the reduced state is the stabilizer state described by the isotropic submodule $M_{I} \subseteq V_{I}$. From (7) we find that

$$
S\left(\rho(M)_{I}\right)=S\left(\rho\left(M_{I}\right)\right)=|I|-\log \left|M_{I}\right| .
$$

The following theorem summarizes the aspects of the phase-space picture of stabilizer states that we will use below to establish our main result. It is stated in such a way as to also apply to the even-dimensional case. Note that the above discussion essentially proves Theorem 1 for odd $d$. We give a general proof in Appendix

Theorem 1 (Stabilizers in phase space). Let $V=\bigoplus_{i=1}^{n} V_{i}=$ $\mathbb{Z}_{d}^{2 n}$ be the phase space for $n$ particles with local dimension $d$, where $d>1$ is an arbitrary integer. There is a one-toone correspondence between isotropic submodules $M \subseteq V$ and equivalence classes $[\rho(M)]$ of stabilizer states on $\left(\mathbb{C}^{\bar{d}}\right)^{\otimes n}$ under conjugation with Weyl operators. Moreover,

$$
\begin{aligned}
{\left[\rho(M)_{I}\right] } & =\left[\rho\left(M_{I}\right)\right], \\
S\left(\left[\rho(M)_{I}\right]\right) & =|I|-\log \left|M_{I}\right| .
\end{aligned}
$$

If $d$ is odd then there is a canonical element $\rho(M)$ in each equivalence class, given by (6). It is compatible with reductions, i.e. $\rho(M)_{I}=\rho\left(M_{I}\right)$.

If the local dimension $d$ is odd, there exists a discrete Wigner function that replicates many properties of its betterknown continuous-variable variant ${ }^{25}$ It is the function on phase space defined by

$$
W_{\rho}(v)=\frac{1}{d^{2 n}} \sum_{v^{\prime} \in V} e^{-\frac{2 \pi \mathrm{i}}{d} 2^{-1}\left[v, v^{\prime}\right]} \operatorname{tr}\left(w\left(v^{\prime}\right)^{\dagger} \rho\right) .
$$

The central observation is that in the case of stabilizer states, the Wigner function $W_{\rho(M)}$ is a probability distribution on 
phase space, i.e. it attains only non-negative values and their sum is one. In fact, $, 25,31$

$$
\begin{aligned}
W_{\rho(M)}(v) & =\frac{1}{d^{2 n}} \sum_{v^{\prime} \in V} e^{-\frac{2 \pi \mathrm{i}}{d} 2^{-1}\left[v, v^{\prime}\right]} \delta_{M}\left(v^{\prime}\right) \\
& =\frac{1}{d^{2 n}} \sum_{v^{\prime} \in M} e^{-\frac{2 \pi \mathrm{i}}{d} 2^{-1}\left[v, v^{\prime}\right]} \\
& =\frac{|M|}{d^{2 n}} \delta_{M^{\perp}}(v) \\
& =\frac{1}{\left|M^{\perp}\right|} \delta_{M^{\perp}}(v),
\end{aligned}
$$

where we have defined the symplectic complement of $M \subseteq V$ by $M^{\perp}=\{v \in V:[v, m]=0 \quad \forall m \in M\}$, for which $|M|\left|M^{\perp}\right|=|V|=d^{2 n}$ and $\left(M^{\perp}\right)^{\perp}=M$. Thus the Wigner function of the stabilizer state with isotropic submodule $M \subseteq$ $V$ is given by the uniform distribution on $M^{\perp} \subseteq V$.

We now show that this construction defines a classical model which reproduces the entropies of the given stabilizer state and its reduced states, up to a certain constant. By phrasing the construction solely in terms of the symplectic complement (hence without recourse to the Wigner function), this result can be established for arbitrary local dimension, even or odd:

Theorem 2 (Classical model for stabilizer states). Let $V=$ $\bigoplus_{i=1}^{n} V_{i}=\mathbb{Z}_{d}^{2 n}$ be the phase space for $n$ particles with local dimension $d$, where $d>1$ is an arbitrary integer. Let $\rho$ be a stabilizer state with isotropic submodule $M \subseteq V$, and define a random variable $X=\left(X_{1}, \ldots, X_{n}\right)$ that takes values uniformly in the symplectic complement $M^{\perp} \subseteq V$. Then,

$$
S\left(\rho_{I}\right)=H\left(X_{I}\right)-|I|
$$

and the same conclusion holds if we replace the Shannon and von Neumann entropy by any Rényi entropy.

If $d$ is odd then the above construction can also be obtained by interpreting the Wigner function $W_{\rho}$ as the probability distribution of the random variable $X$.

Proof. To prove 11, denote by $\pi_{I}: V \rightarrow V_{I}$ the projection onto the phase space of parties $I \subseteq\{1, \ldots, n\}$. It will be convenient to consider $V_{I}$ as a submodule of $V$ in the natural way. To avoid any notational ambiguity, we denote by $X^{\perp_{I}}$ the symplectic complement of a subspace $X$ taken within $V_{I}$.

Observe that

$$
\pi_{I}\left(M^{\perp}\right) \subseteq M_{I}^{\perp_{I}}
$$

Indeed, if $v \in M^{\perp}$ and $m_{I} \in M_{I}$, then $\left[\pi_{I}(v), m_{I}\right]=$ $\left[v, m_{I}\right]=0$. On the other hand, we find that

$$
\pi_{I}\left(M^{\perp}\right)^{\perp_{I}} \subseteq M_{I}
$$

To see this, consider a vector $v_{I} \in V_{I}$ and note that if $v_{I} \perp$ $\pi_{I}\left(M^{\perp}\right)$ then $v_{I} \perp M^{\perp}$, hence $v_{I} \in M \cap V_{I}=M_{I}$ since $\left(M^{\perp}\right)^{\perp}=M$. We conclude from (12) and 13 that

$$
\pi_{I}\left(M^{\perp}\right)=M_{I}^{\perp_{I}}
$$

Note that $X_{I}=\pi_{I}(X)$. Since $\pi_{I}$ is a group homomorphism, it follows that $X_{I}$ is distributed uniformly on its range, so that

$$
\begin{aligned}
H\left(X_{I}\right) & =\log \left|\pi_{I}\left(M^{\perp}\right)\right|=\log \left|M_{I}^{\perp_{I}}\right|=\log \frac{d^{2|I|}}{\left|M_{I}\right|} \\
& =2|I|-\log \left|M_{I}\right|=|I|+S\left(\rho_{I}\right),
\end{aligned}
$$

where we have used 9 in the last step. We have thus established 11].

The same result holds if we replace the Shannon and von Neumann entropy by Rényi entropies. This is because the stabilizer states $\rho(M)_{I}$ are normalized projectors and each random variable $X_{I}$ is distributed uniformly on its range, so that the entropies coincide.

Finally, it is clear from (10) that for odd $d$ the distribution of $X$ coincides with the Wigner function $W_{\rho(M)}$ of the stabilizer state. It remains to show that the Wigner function $W_{\rho_{I}}$ of a reduced state $\rho_{I}$ is obtained by marginalizing the full Wigner function (in other words: the quantum and the classical way of reducing to subsystems commute):

$$
W_{\rho_{I}}(v)=\sum_{w: w_{I}=v} W_{\rho}(w)
$$

for all $v \in V_{I}$. While this can easily be proved in full generality from the definition of the Wigner function, it is also true that for the special case of stabilizer states, Eq. (15) follows directly from (14).

Corollary 3. Stabilizer states satisfy all balanced information inequalities. Moreover, they satisfy the Ingleton inequality (3).

Proof. As described in the introduction, the first claim follows immediately from (11). This is because for any balanced information inequality $\sum_{I} \nu_{I} H\left(X_{I}\right) \geq 0$ we necessarily have that ${ }^{18}$

$$
\sum_{I} \nu_{I}|I|=\sum_{I}\left(\sum_{i \in I} \nu_{I}\right)=\sum_{i}\left(\sum_{I \ni i} \nu_{I}\right)=0 .
$$

Hence the correction term in 111 cancels as we sum over all subsystems:

$$
\sum_{I} \nu_{I} S\left(\rho_{I}\right)=\sum_{I} \nu_{I} H\left(X_{I}\right)-\sum_{I} \nu_{I}|I| \geq 0 .
$$

For the second claim, we note that Ref. 15 shows that the Ingleton inequality (3) holds for the random variables $X_{I}=\pi_{I}(X)$. In the language of Ref. 14, this is because the entropy vector $\left(H\left(X_{I}\right)\right)$ can be characterized by the normal subgroups $\operatorname{ker}\left(\pi_{I}\right) \cap M^{\perp}$ (in fact, our phase spaces are even Abelian groups). Since the Ingleton inequality is balanced, the argument given above shows that it also holds for the von Neumann entropies of stabilizer states.

Pure stabilizer states correspond to maximally isotropic submodules $M \subseteq V$. Such submodules are called Lagrangian, and they satisfy $|M|=d^{n}$ and $M=M^{\perp}$. Thus in this case our classical model can also be defined by choosing 
$X \in M$ uniformly at random. Furthermore, since $\pi_{I}(M) \cong$ $M /\left(\operatorname{ker} \pi_{I} \cap M\right)$, we may also define $X_{I}$ to be the coset of $X$ modulo $\operatorname{ker}\left(\pi_{I}\right) \cap M=M \cap V_{I^{c}}=M_{I^{c}}$. In this way, we recover the construction of Theorem 11 in Ref. 1 .

\section{GAUSSIAN STATES}

We sketch the corresponding result for Gaussian states of continuous-variable systems. The Wigner function of an $n$ mode Gaussian quantum state $\rho$ with covariance matrix $\Sigma$ and first moments $\mu$ is defined as follows on classical phase space $\mathbb{R}^{2 n}$ :

$$
W_{\rho}(x)=\frac{1}{(2 \pi)^{n}(\operatorname{det} \Sigma)^{\frac{1}{2}}} e^{-\frac{1}{2}(x-\mu)^{T} \Sigma^{-1}(x-\mu)},
$$

(see e.g. the review Ref. 32). Evidently, $W_{\rho}$ is the probability density of a random vector $X=\left(X_{1}, \ldots, X_{2 n}\right)$ with multivariate normal distribution of mean $\mu$ and covariance matrix $\Sigma$. Using the well-known relation $\operatorname{tr} \rho^{2}=(2 \pi)^{n} \int W_{\rho}^{2}(x) d x$, it follows that the Rényi-2 entropy of the quantum state, $S_{2}(\rho)=-\log \operatorname{tr} \rho^{2}$, is directly related to the differential Rényi-2 entropy of the random variable $X, H_{2}(X)=$ $-\log \int W_{\rho}^{2}(x) d x$ :

$$
S_{2}(\rho)=H_{2}(X)-n \log (2 \pi) .
$$

The reduced state $\rho_{I}$ for some subset of modes $I \subseteq$ $\{1, \ldots, n\}$ is again a Gaussian state, and its covariance matrix is equal to the corresponding submatrix of $\Sigma$. Thus the Wigner function of $\rho_{I}$ is given by the marginal probability density of the variables $X_{I}=\left(X_{i}\right)_{i \in I}$, and using (16) we find that

$$
S_{2}\left(\rho_{I}\right)=H_{2}\left(X_{I}\right)-|I| \log (2 \pi) .
$$

Equation (17) states that the Rényi-2 entropy of a Gaussian quantum state is always lower than the phase space entropy of its classical model, as given by the Wigner function. It is so by a precise amount, namely by $\log (2 \pi)$ bits per mode.

Theorem 4 (Classical model for Gaussian states). Let $\rho$ be a Gaussian state with covariance matrix $\Sigma$, and define a random variable $X=\left(X_{1}, \ldots, X_{n}\right)$ with probability density given by the Wigner function $W_{\rho}(x)$. Then, for any positive $\alpha \neq 1$,

$$
S_{2}\left(\rho_{I}\right)=H_{\alpha}\left(X_{I}\right)-|I|\left(\log \pi-\frac{\log \alpha}{1-\alpha}\right),
$$

where $H_{\alpha}(X)=1 /(1-\alpha) \log \int W_{\rho}^{\alpha}(x) d x$ is the differential Rényi- $\alpha$ entropy. In the limit $\alpha \rightarrow 1$, we recover

$$
S_{2}\left(\rho_{I}\right)=H\left(X_{I}\right)-|I|(\log \pi+1) .
$$

where $H(X)=-\int W_{\rho}(x) \log W_{\rho}(x) d x$ is the differential Shannon entropy.

Proof. By Gaussian integration, the differential Rényi- $\alpha$ entropy of the random variable $X_{I}$ is given by

$$
H_{\alpha}\left(X_{I}\right)=\frac{1}{2} \log \operatorname{det} \Sigma+|I|\left(\log 2 \pi-\frac{\log \alpha}{1-\alpha}\right) .
$$

The assertions of the theorem follow from this and (17).
Equation (18) has been previously used in Ref. 16, where the formula is attributed to Stratonovich. Just as in the discrete case, we immediately get the following corollary:

Corollary 5. The Rényi-2 entropy for Gaussian states satisfies all balanced information inequalities that are valid for multivariate normal distributions.

Interestingly, Gaussian states can violate the Ingleton inequality (as opposed to stabilizer states, cf. Corollary 3). Indeed, this is well-known for multivariate normal distributions, and it is readily verified that the counterexample presented in Ref. 18 is a physical covariance matrix (i.e., it satisfies the uncertainty relation $\Sigma+i \Omega \geq 0$, where $\Omega$ is the symplectic matrix). Thus, by Theorem 4 the corresponding Gaussian state violates the Ingleton inequality.

\section{ACKNOWLEDGEMENTS}

We would like to thank Matthias Christandl for many fruitful discussions. This work has benefited from insightful comments of an anonymous referee and the associate editor. DG's research is supported by the Excellence Initiative of the German Federal and State Governments (ZUK 43). MW acknowledges support of the Swiss National Science Foundation (PP00P2-128455), the German Science Foundation (CH 843/2-1), and the National Center of Competence in Research 'Quantum Science and Technology'.

\section{Appendix A: Phase Space Approach to Stabilizer States}

In this appendix, we present a self-contained account of Weyl operators and stabilizer states in the discrete phase-space picture. This section does not contain original results. All statements could be found in some form in Refs. 25, 29, 3335, albeit not in a unified language.

Discrete symplectic geometry. Let $d>1$ be an integer and let $\mathbb{Z}_{d}=\mathbb{Z} / d \mathbb{Z}$ be the congruence classes of integers modulo $d$. The phase space for $n$ particles with local dimension $d$ is by definition $V=\bigoplus_{i=1}^{n} V_{i}=\mathbb{Z}_{d}^{2 n}$, the free $\mathbb{Z}_{d}$-module of rank $2 n$. Given a point $v \in V$, we write $v_{i}=\left(p_{i}, q_{i}\right) \in V_{i}=\mathbb{Z}_{d}^{2}$ for its components. Consider the bilinear form $[-,-]: V \times$ $V \rightarrow \mathbb{Z}_{d}$ defined by

$$
\left[v, v^{\prime}\right]=\sum_{i=1}^{n} p_{i} q_{i}^{\prime}-q_{i} p_{i}^{\prime} .
$$

It is non-degenerate and totally isotropic, i.e. $[v, v]=0$ for all $v \in V$. If $d$ is a prime then the phase space $V$ is simply a symplectic vector space over the finite field $\mathbb{F}_{d}=\mathbb{Z}_{d}$. We will also in the general case refer to $[-,-]$ as the symplectic form.

A character of a finite Abelian group $G$ is a homomorphism $G \rightarrow U(1)$. Denote by $\widehat{G}$ the set of characters, which is again an Abelian group with the operation of pointwise multiplication. It is called the (Pontryagin) dual of $G$. It is well-known 
that $G \cong \hat{G}$, although not canonically. For the cyclic group $G=\mathbb{Z}_{d}$, all characters are powers of $\chi_{d}(x)=e^{\frac{2 \pi \mathrm{i}}{d} x}$.

Lemma 6. The characters of the additive group of the phase space $V$ are $\hat{V}=\left\{\chi_{d}([v,-]): v \in V\right\} \cong V$.

Proof. By injectivity of $\chi_{d}$ and non-degeneracy of the symplectic form, each $v$ determines a different character. Thus we have found all $|\hat{V}|=|V|$ many characters.

The symplectic complement of a submodule $M \subseteq V$ is the submodule $M^{\perp}=\{v \in V:[v, m]=0 \forall m \in M\}$. In the case of prime $d$, it is well-known that $\operatorname{dim} M+\operatorname{dim} M^{\perp}=$ $\operatorname{dim} V$-however, for general submodules the dimension (or rank) might not even be well-defined. Still there is an important analogue that holds in the general case:

Lemma 7. $|M|\left|M^{\perp}\right|=|V|$.

Proof. We show that the group homomorphism

$$
\Phi: M^{\perp} \rightarrow \widehat{V / M}, \quad v \mapsto\left([w] \mapsto \chi_{d}([v, w])\right) .
$$

is both injective and surjective (it is certainly well-defined). Injectivity follows immediately from the non-degeneracy of the symplectic form. For surjectivity, let $\tau \in \widehat{V / M}$. Then $w \mapsto \tau([w])$ is a character of $V$. By Lemma 6 there exists $v \in V$ such that $\tau([w])=\chi_{d}([v, w])$. Since $\tau$ vanishes on $M$, $v \in M^{\perp}$. Thus $\Phi$ is an isomorphism, and we find that

$$
\left|M^{\perp}\right|=|\widehat{V / M}|=|V / M|=\frac{|V|}{|M|} .
$$

The following important corollary follows from Lemma 7 and $M \subseteq\left(M^{\perp}\right)^{\perp}$.

Corollary 8. $\left(M^{\perp}\right)^{\perp}=M$.

We call a submodule $M \subseteq V$ an isotropic submodule if $M \subseteq M^{\perp}$, i.e. if $\left[m, m^{\prime}\right]=0$ for all $m, m^{\prime} \in M$.

Finally, consider $V=\bigoplus_{i \in I} V_{i}$, the phase space of particles $I \subseteq\{1, \ldots, n\}$. There is a natural way of restricting a submodule $M$ to $V_{I}$ : we set

$$
M_{I}:=M \cap V_{I},
$$

where $V_{I}$ is identified with a submodule of $V$ in the natural way.

Weyl representation. Following Refs. 33 and 34 , we first define Weyl operators for general integers $(P, Q) \in \mathbb{Z}^{2}$, not necessarily in the range $\{0, \ldots, d-1\}$. These are the unitaries on $L^{2}\left(\mathbb{Z}_{d}\right) \cong \mathbb{C}^{d}$ given by

$$
(W(P, Q) \psi)(x)=\tau_{2 d}(-P Q) \chi_{d}(P x) \psi(x-Q),
$$

where $\tau_{2 d}(R)=\chi_{2 d}\left(\left(d^{2}+1\right) R\right)$. For example, $W(1,0)$ is the $Z$-operator $|x\rangle \mapsto e^{\frac{2 \pi \mathrm{i}}{d} x}|x\rangle$, while $W(0,1)$ is the $X$-operator $|x\rangle \mapsto|x+1(\bmod d)\rangle$. By direct computation, 34

$$
\begin{aligned}
& W(P, Q) W\left(P^{\prime}, Q^{\prime}\right) \\
= & \tau_{2 d}\left(P Q^{\prime}-Q P^{\prime}\right) W\left(P+P^{\prime}, Q+Q^{\prime}\right), \\
& W(P, Q)^{-1} \\
= & W(P, Q)^{\dagger}=W(-P,-Q), \\
& W(P, Q) W\left(P^{\prime}, Q^{\prime}\right) \\
= & \chi_{d}\left(P Q^{\prime}-Q P^{\prime}\right) W\left(P^{\prime}, Q^{\prime}\right) W(P, Q) .
\end{aligned}
$$

We now introduce the Weyl operators $w(p, q)$ for congruence classes $(p, q) \in \mathbb{Z}_{d}^{2}$. It is here that the treatment of the odd and the even-dimensional case diverges.

For $d$ odd, $\tau_{2 d}(1)=\chi_{d}\left(\frac{d^{2}+1}{2}\right)$ is a $d$-th root of unity, so that $W(P+d, Q)=W(P, Q+d)=W(P, Q)$. In other words, $W$ is constant on congruence classes modulo $d$, so we can directly define $w(p, q):=W(P, Q)$. In fact, $2^{-1}:=\frac{d^{2}+1}{2} \in \mathbb{Z}$ is the multiplicative inverse of 2 modulo $d$, so that we recover the formulas from Section

$$
\begin{array}{r}
(w(p, q) \psi)(x)=\chi_{d}\left(p x-2^{-1} p q\right) \psi(x-q) \\
w(v) w\left(v^{\prime}\right)=\chi_{d}\left(2^{-1}\left[v, v^{\prime}\right]\right) w\left(v+v^{\prime}\right) .
\end{array}
$$

For $d$ even, $\tau_{2 d}(1)=\chi_{2 d}(1)$ is a primitive $2 d$-th root of unity (e.g., in the case of qubits $\tau_{2 d}(1)=i$ ). Equation A1 then implies that $W(P+d, Q)$ and $W(P, Q+d)$ are either $W(P, Q)$ or $-W(P, Q)$. In order to fix the sign, we choose $w(p, q):=W(P, Q)$, where $(P, Q)$ is the unique preimage in $\{0, \ldots, d-1\}^{2} \subseteq \mathbb{Z}^{2}$. Because $w$ and $W$ differ at most by a phase, A1 still implies that $(p, q) \mapsto w(p, q)$ defines a projective representation of the (additive structure of the) phase space $\mathbb{Z}_{d}^{2}$.

In both the even and the odd case, it now follows from (A2) and $\mathrm{A} 3$ that

$$
\begin{aligned}
w(v)^{-1} & =w(v)^{\dagger}= \pm w(-v), \\
w(v) w\left(v^{\prime}\right) & =\chi_{d}\left(\left[v, v^{\prime}\right]\right) w\left(v^{\prime}\right) w(v) .
\end{aligned}
$$

For $n$-particles, the phase space is the direct sum $V=$ $\bigoplus_{i=1}^{n} V_{i}=\mathbb{Z}_{d}^{2 n}$. We define its Weyl representation on $\left(\mathbb{C}^{d}\right)^{\otimes n}$ by the tensor product of the single-particle representations, $w(v)=\bigotimes_{i=1}^{n} w\left(v_{i}\right)$. In this way, the relations and (A6) continue to hold. Moreover, it is easy to verify that

$$
\operatorname{tr} w(v)=d^{n} \delta_{v, 0}
$$

Stabilizer states. To define stabilizer states, we start with a stabilizer group $G$, i.e. a finite Abelian group whose elements are multiplies of Weyl operators on $\left(\mathbb{C}^{d}\right)^{\otimes n}$, such that the only multiple of $\mathbb{1}=w(0)$ contained in $G$ is $\mathbb{1}$ itself. With such a group we associate the operator

$$
P=\frac{1}{|G|} \sum_{g \in G} g .
$$


From the fact that $G$ is a group, we deduce that $P^{2}=P$; since all elements of $G$ are unitaries, $P=P^{\dagger}$; and A7 implies that $\operatorname{tr} P=d^{n} /|G|$. Hence $P$ projects onto a $\left(d^{n} /|G|\right)$ dimensional subspace, called the stabilizer code of $G$. The corresponding stabilizer state is $\rho=\frac{1}{d^{n}} \sum_{g} g$. We now prove Theorem 1 which we repeat for the reader's convenience:

Theorem 1(Stabilizers in phase space). Let $V=\bigoplus_{i=1}^{n} V_{i}=$ $\mathbb{Z}_{d}^{2 n}$ be the phase space for $n$ particles with local dimension $d$, where $d>1$ is an arbitrary integer. There is a one-toone correspondence between isotropic submodules $M \subseteq V$ and equivalence classes $[\rho(M)]$ of stabilizer states on $\left(\mathbb{C}^{\bar{d}}\right)^{\otimes n}$ under conjugation with Weyl operators. Moreover,

$$
\begin{aligned}
{\left[\rho(M)_{I}\right] } & =\left[\rho\left(M_{I}\right)\right], \\
S\left(\left[\rho(M)_{I}\right]\right) & =|I|-\log \left|M_{I}\right| .
\end{aligned}
$$

If $d$ is odd then there is a canonical element $\rho(M)$ in each equivalence class, given by (6). It is compatible with reductions, i.e. $\rho(M)_{I}=\rho\left(M_{I}\right)$.

Proof. 1. From isotropic submodules to classes of stabilizer states: Let $M \subseteq \mathbb{Z}_{d}^{2 n}$ be an isotropic submodule. Since $M$ is a finite Abelian group, it can be written as a direct sum of cyclic groups, $M \cong \mathbb{Z}_{d_{1}} \oplus \ldots \oplus \mathbb{Z}_{d_{k}}$. Let $m_{j} \in M$ be a generator of the $j$-th cyclic subgroup. Since $w\left(m_{j}\right)^{d_{j}} \propto w(0)=\mathbf{1}$, we can choose phases $\lambda_{j}$ such that $\left(\lambda_{j} w\left(m_{j}\right)\right)^{d_{j}}=1$. Define

$$
G=\{\underbrace{\prod_{j=1}^{k}\left(\lambda_{j} w\left(m_{j}\right)\right)^{x_{j}}}_{=: \mu_{m} w(m)}: m=\sum_{j} x_{j} m_{j} \in M\} .
$$

The product is well-defined, because by $\mathrm{A6}$, the Weyl operators $\{w(m): m \in M\}$ all commute. Thus the data $M, \mu$ define a stabilizer group of cardinality $|M|$ with corresponding stabilizer state $\rho(M, \mu)=\frac{1}{d^{n}} \sum_{m \in M} \mu_{m} w(m)$. This state depends on the phases $\mu_{m}$, which in turn resulted from our choice of generators $m_{j}$ and phases $\lambda_{j}$. A different choice would have resulted in another stabilizer group $\left\{\nu_{m} w(m) \mid m \in M\right\}$ and we have yet to show that the two groups are related by conjugation with some Weyl operator. To this end, note that A7 implies that necessarily $\nu_{m} w(m) \nu_{m^{\prime}} w\left(m^{\prime}\right)=\nu_{m+m^{\prime}} w\left(m+m^{\prime}\right)$. It follows that $\tau(m):=\nu_{m} / \mu_{m}$ defines a character of $M$. Since $V$ is an Abelian group, this character can be extended to all of $V$, and it is therefore by Lemma 6 of the form $\tau(m)=\chi_{d}([v, m])$ for some $v \in V$. But then it follows from that

$$
w(v) \mu_{m} w(m) w(v)^{\dagger}=\tau(m) \mu_{m} w(m)=\nu_{m} w(m) .
$$

Consequently, $\rho(M, \nu)$ and $\rho(M, \mu)$ are related by conjugation with the Weyl operator $w(v)$.

If $d$ is odd, then $\mathrm{A} 4$ implies that $w(m) w\left(m^{\prime}\right)=w(m+$ $\left.m^{\prime}\right)$. It follows that $G:=\{w(m): m \in M\}$ is a stabilizer group of cardinality $|M|$, with corresponding stabilizer state $\rho(M)=\frac{1}{d^{n}} \sum_{m \in M} w(m)$. This is the canonical representative (6) of the equivalence class of states associated with $M$.

2. Surjectivity: Here, we show that our map from isotropic submodules to equivalence classes of stabilizer states is surjective, Let $G$ be a stabilizer group with corresponding stabilizer state $\rho$. Equation A7 implies that, for each $g \in G$ there exists a unique $m_{g} \in V$ such that $g \propto w\left(m_{g}\right)$. Conversely, no two $m_{g}$ can be equal- otherwise, two group elements in $G$ would differ only by a phase and hence there would be a non-trivial multiple of $\mathbb{1}$ in $G$. Define $M:=\left\{m_{g}\right\}$. Then $M$ is a submodule of $V$, since $m_{g}+m_{h}=m_{g h}$. Since $G$ is Abelian, all $w\left(m_{g}\right)$ commute and s6ows that $M$ is isotropic. Then $M$ is indeed a preimage of $[\rho]$, since by its very construction there exists a choice of phases by which we recover $G$ (namely $\mu_{m_{g}}=g w\left(m_{g}\right)^{-1}$ ).

3. Injectivity: Suppose that $\rho(M, \mu)$ and $\rho\left(M^{\prime}, \mu^{\prime}\right)$ are two equivalent stabilizer states. As we saw, conjugating with a Weyl operator only changes the phases, so we may in fact assume that states are equal. Now assume that $M \neq M^{\prime}$, so that there exists e.g. $m \in M \backslash M^{\prime}$. Then, A7 shows that

$$
0 \neq \operatorname{tr} w(m) \rho(M, \mu)=\operatorname{tr} w(m) \rho\left(M^{\prime}, \mu^{\prime}\right)=0,
$$

which is the desired contradiction.

4. Reduction: We now show that our construction is compatible with reduction. For this, observe that

$$
\operatorname{tr}_{I^{c}} w(m)=\left(\prod_{i \in I} w\left(m_{i}\right)\right) d^{|I|^{c}} \delta_{m \in M_{I}} .
$$

Since any valid assignment of phases $\mu_{m}$ restricts to the submodule $M_{I}=M \cap V_{I}$, it follows that $\left[\rho(M)_{I}\right]=\left[\rho\left(M_{I}\right)\right]$. It is also immediate that the canonical element (6) is compatible with reduction.

5. Entropy: In view of the last point, it suffices to show the statement about entropies for $I=\{1, \ldots, n\}$. Recall that the cardinality of $M$ and of the corresponding stabilizer groups $G$ agree. We have already seen that the dimension of the stabilizer code is equal to $d^{n} /|G|$. Thus,

$$
S([\rho(M)])=n-\log |G|=n-\log |M| .
$$

${ }^{1}$ N. Linden, M. B. Ruskai, and A. Winter, "The Quantum Entropy Cone of Stabiliser States," arXiv:1302.5453 (2013).

${ }^{2}$ N. Pippenger, "What are the laws of information theory?" Spec. Prob. on Comm. Comp. Conf., Palo Alto (1986).

${ }^{3}$ R. W. Yeung, "A framework for linear information inequalities," IEEE Trans. Inf. Theory 43, 1924-1934 (1997).

${ }^{4}$ Z. Zhang and R. W. Yeung, "On Characterization of Entropy Function via Information Inequalities," IEEE Trans. Inf. Theory 44, 1440-1452 (1998).

${ }^{5}$ F. Matus, "Infinitely many information inequalities," Proc. Int. Symp. Inf. Theory (ISIT) , 41-44 (2007).

${ }^{6}$ M. A. Nielsen and I. L. Chuang, Quantum Computation and Quantum Information (Cambridge University Press, 2004).

${ }^{7}$ N. Pippenger, "The Inequalities of Quantum Information Theory," IEEE Trans. Inf. Theory 49, 773-789 (2003).

${ }^{8}$ E. H. Lieb and M. B. Ruskai, "Proof of the Strong Subadditivity of Quantum-Mechanical Entropy,” J. Math. Phys. 14, 1938-1941 (1973).

${ }^{9}$ N. Linden and A. Winter, "A New Inequality for the von Neumann Entropy," Commun. Math. Phys. 259, 129-138 (2005).

${ }^{10}$ J. Cadney, N. Linden, and A. Winter, "Infinitely Many Constrained Inequalities for the von Neumann Entropy," IEEE Trans. Inf. Theory 58, 3657-3663 (2012).

${ }^{11}$ T. S. Han, "Linear dependence structure of the entropy space," Information and Control 29, 337 (1975).

${ }^{12}$ T. H. Chan, "Balanced Information Inequalities," IEEE Trans. Inf. Theory 49, 3261 (2003).

${ }^{13}$ N. Linden, M. Mosonyi, and A. Winter, "The structure of Rényi entropic inequalities," arXiv:1212.0248 (2012). 
${ }^{14}$ T. H. Chan and R. W. Yeung, "On a Relation Between Information Inequalities and Group Theory,” IEEE Trans. Inf. Theory 48, 1992-1995 (2002).

${ }^{15} \mathrm{H}$. Li and E. K. P. Chong, "On Connections between Group Homomorphisms and the Ingleton Inequality," in Proc. Int. Symp. Inf. Theory (ISIT) (2007).

${ }^{16}$ G. Adesso, D. Girolami, and A. Serafini, "Measuring Gaussian Quantum Information and Correlations Using the Rényi Entropy of Order 2," Phys. Rev. Lett. 109, 190502 (2012).

${ }^{17}$ O. Holtz and B. Sturmfels, "Hyperdeterminantal relations among symmetric principal minors," J. Algebra 316, 634-648 (2007).

${ }^{18}$ S. Shadbakht and B. Hassibi, "On the Entropy Region of Gaussian Random Variables," arXiv:1112.0061 (2011).

${ }^{19}$ D. Gross and J. Eisert, "Quantum Margulis expanders," Quant. Inf. Comp. 8, 722 (2008).

${ }^{20}$ V. Veitch, C. Ferrie, D. Gross, and J. Emerson, "Negative quasi-probability as a resource for quantum computation," New J. Phys. 14, 113011 (2012).

${ }^{21}$ A. Mari and J. Eisert, "Positive Wigner Functions Render Classical Simulation of Quantum Computation Efficient," Phys. Rev. Lett. 109, 230503 (2012).

${ }^{22}$ V. Veitch, N. Wiebe, C. Ferrie, and J. Emerson, "Efficient simulation scheme for a class of quantum optics experiments with non-negative Wigner representation," New J. Phys. 15, 013037 (2013).

${ }^{23}$ M. Howard, V. Veitch, and J. Emerson, In preparation (2013).

${ }^{24} \mathrm{R}$. Hudson, "When is the Wigner quasi-probability density non-negative?" Rep. Math. Phys. 6, 249 (1974).
${ }^{25}$ D. Gross, "Hudson's Theorem for finite-dimensional quantum systems," J. Math. Phys. 47, 122107 (2006).

${ }^{26} \mathrm{D}$. Gross, "Non-negative Wigner functions in prime dimensions," Appl. Phys. B 86, 367 (2007).

${ }^{27}$ M. Christandl and G. Mitchison, "The Spectra of Quantum States and the Kronecker Coefficients of the Symmetric Group," Commun. Math. Phys. 261, 789-797 (2006).

${ }^{28}$ M. Christandl, M. B. Şahinoğlu, and M. Walter, "Recoupling Coefficients and Quantum Entropies," arXiv:1210.0463 (2012).

${ }^{29} \mathrm{D}$. Gottesman, Stabilizer codes and quantum error correction, Ph.D. thesis, Caltech (1996).

${ }^{30}$ G. Folland, Harmonic Analysis in Phase Space (Princeton University Press, 1989).

${ }^{31} \mathrm{D}$. Gross, "Finite phase space methods in quantum information," Diploma thesis, University of Potsdam (2005).

${ }^{32}$ C. Weedbrock, S. Pirandola, R. García-Patrón, N. J. Cerf, T. C. Ralph, J. H. Shapiro, and S. Lloyd, "Gaussian Quantum Information," Rev. Mod. Phys. 84, 621-669 (2012).

${ }^{33} \mathrm{D}$. Appleby, "Symmetric informationally complete-positive operator valued measures and the extended Clifford group," J. Math. Phys. 46, 052107 (2005).

${ }^{34} \mathrm{~N}$. Beaudrap, "A linearized stabilizer formalism for systems of finite dimension,” Quant. Inf. Comp. 13, 73 (2013).

${ }^{35}$ R. Kueng and D. Gross, In preparation (2013). 